\title{
Vivir con VIH: experiencias de estigma sentido en personas con
}

\section{(1)PSicogente ISSN 0124-0137 EISNN 2027-212X}

\section{Artículo de Investigación} Copyright 2018 by Psicogente

\section{Correspondencia de autores:} yguevara@javerianacali.edu.co paulahoyos@javerianacali.edu.co

Recibido: 03-02-2017

Aceptado: $30-05-2017$

Publicado: 01-01-18

\section{Living with HIV: Stigmatizing experiences in people with HIV}

\author{
Yurany Guevara-Sotelo ${ }^{(D)}$ y Paula Andrea Hoyos-Hernández \\ Pontificia Universidad Javeriana, Cali, Colombia
}

Resumen

Objetivo: Reconocer los significados colectivos y subjetivos que un grupo de personas con VIH tiene respecto a vivir con la enfermedad.

Método: Cualitativo explicativo con diseño narrativo. Los datos fueron recolectados a través de tres grupos de discusión conformados por 16 personas con VIH, hombres y mujeres entre los 22 y los 49 años de edad, que viven en la ciudad de Cali.

Resultados: Los significados de los participantes giran en torno al vivir el VIH, en relación con el estigma sentido, el estigma real, el estigma percibido y el autoestigma. La experiencia del estigma se relaciona directamente con la percepción que los participantes tienen de sí mismos y la construcción de su autoimagen y autoconcepto se transforma dependiendo de la forma en que se asume la enfermedad; finalmente, las estrategias de afrontamiento que se utilizan se basan en el cuidado de la imagen, en evitar el malestar emocional y sentirse apoyados por alguna persona cercana a ellos.

Conclusiones: Los hallazgos de la presente investigación permiten evidenciar que el VIH es producto de diversas situaciones en cadena, en donde las representaciones sociales construidas alrededor de la enfermedad constituyen un factor central en el afrontamiento y en la forma de vivirlo colectivamente, realizar una intervención desde temáticas como la memoria colectiva, la identidad colectiva y la reconstrucción de la identidad podría permitir resignificar y potencializar la imagen que las personas con $\mathrm{VIH}$ tienen de sí mismas y del grupo social al que pertenecen.

Palabras Claves:

Estigma sentido, VIH, Autoimagen, Identidad colectiva

\section{Abstract}

Objective: This paper aims to recognize what a group of people with HIV have regarding living with this disease and based on collective and subjective meanings.

Method: The data collected corresponds to three discussion groups of 16 people with HIV, men and women from Cali, between 22 and 49 years sampled, through a Qualitative explanatory and following a narrative design

Results: As a result, everything related to collective meanings of the participants revolve around living with this disease (HIV), as follows: Felt stigma, real stigma, perceived stigma and self-stigma; in addition, the experience of stigma is directly related to the participants perception of themselves and the construction of their self-image and self-concept, transformed depending on how do these individuals assume the disease; finally, coping strategies used are based on caring for the image, avoiding emotional distress and feeling supported by someone close to them.

Conclusion: This research findings make possible to show that HIV is the result of various situations associated, where social representations built around the disease are a central factor in coping with and how to live with it collectively. Themes such as collective memory, collective identity and the reconstruction of identity could make possible to reframe and enhance the image that people with HIV have of themselves and the social group to which they belong to as well.

Key words: Military, Civilians, Attitudes, Demobilized. 


\section{Karen Macías Pulgarín, Paula Andrea Mendoza, Christian León Osorio, Fernando Riveros Munévar, Anderssen Vera, Arleth Patricia Bernal, Liliana Reyes}

Como citar este artículo (APA):

Guevara-Sotelo, Y. \& Hoyos-Hernández, P.A. (2018). Vivir con VIH: experiencias de estigma sentido en personas con VIH. Psicogente, 21(39), 127-139. http://doi.org/10.17081/psico.21.39.2827

\section{INTRODUCCIÓN}

El VIH es una enfermedad que desde su aparición ocasionó millones de muertes de personas en el mundo a causa del SIDA. Esta llegó a convertirse en una pandemia de gran preocupación para las organizaciones mundiales que trabajan el tema de la salud, generando movilizaciones en diferentes ámbitos orientados a controlar la morbimortalidad a causa de esta (Organización Mundial de la Salud [OMS], 2011). Sin embargo, la huella social que ha dejado el VIH es de gran importancia analizarla. Se estima que esta se relaciona con el estigma social, el cual se caracteriza por ser una descalificación que utilizan un grupo de individuos para señalar a otros como personas con un bajo valor social que no tienen poder para acceder a la calidad de vida adecuada (Goffman, 1963).

Se puede describir a manera de hipótesis que el estigma social respecto al VIH aparece como un producto de la clasificación moral que en el siglo XX las instituciones políticas y religiosas hacían a las personas que adquirieron esta enfermedad que en su momento fue enigmática, sin explicación y sin tratamiento, así mismo, asociando esta condición a grupos, que a nivel social tenían un papel negativo: los homosexuales, quienes ejercen el trabajo sexual y los consumidores de sustancias psicoactivas inyectables (Gallo \& Montagnier, 2004; Hoyos-Hernández \& Duarte-Alarcón, 2016).

En este orden de ideas, se debe destacar que, aunque el VIH es una enfermedad de características principalmente biológicas, es posible evidenciar tiene unas implicaciones psicosociales que pueden llegar a ocasionar dificultades en la dimensión psicológica de un individuo, quien se empieza a identificar desde el prejuicio real o imaginario que la sociedad ha construido al respecto y ocasiona malestar emocional. A este último se le denomina estigma sentido, el cual se divide en estigma percibido y autoestigma; el estigma percibido se refiere a las anticipaciones que una persona con VIH hace a partir de las conductas negativas reales o imaginarias que la sociedad haya tenido hacia ella; el autoestigma tiene que ver con los comportamientos negativos como el rechazo, la repulsión y la subestimación que una persona con VIH tiene a sí misma por percibir de manera real o imaginaria prejuicios negativos hacia ella por tener la enfermedad (Moral \& Segovia, 2015; Jiménez et al., 2012; Miric, 2004).

Teniendo en cuenta el VIH como problemática social, esta enfermedad se puede convertir en una cadena de situaciones: el VIH conlleva al estigma social, el estigma social conlleva al estigma sentido, el estigma sentido conlleva al autoestigma, el autoestigma puede producir la construcción de la identidad con base en la enfermedad como fenómeno rechazado socialmente; esta construcción de identidad desde lo que significa la enfermedad y no lo que significa la subjetividad puede conllevar a un malestar a nivel general, afectar la autoimagen, el autoconcepto, impactar negativamente en la adherencia al tratamiento, la pérdida de apoyo social y disminuir la posibilidad de una persona de tener una adecuada calidad de vida. 


\section{Karen Macías Pulgarín, Paula Andrea Mendoza, Christian León Osorio, Fernando Riveros Munévar, Anderssen Vera, Arleth Patricia Bernal, Liliana Reyes}

Un estudio realizado en República Dominicana aplicó un instrumento para detectar el grado de estigma sentido y su relación con otros factores a una población de 212 personas con VIH. En él se encontró correlaciones significativamente altas entre los niveles del estigma sentido y las medidas de autoestima ( $p=.010)$; además, se presentaba correlación baja entre percepción de apoyo social y depresión, lo que dio a conocer que el aspecto psicosocial estaba relacionado con el estigma sentido de las personas que vivían con el VIH/SIDA (Miric, 2004). Por otro lado, en Puerto Rico se realizó un estudio con una población de 249 personas ( $59 \%$ hombres) a los cuales se aplicó la Escala de Estigma Sentido para Personas con VIH (HIV Felt Stigma Scale, 2010). De la población encuestada el 80 \% tenía algún grado de estigma sentido, de los cuales 40 \% representó niveles moderados y severos de estigma sentido (Jiménez et al., 2010).

La presente investigación tuvo como objetivo reconocer y caracterizar en un grupo de personas con $\mathrm{VIH}$, las percepciones subjetivas y colectivas de lo que significa vivir con la enfermedad. Esta se fundamentó en la teoría de la identidad social y la categorización del yo (Tajfel, 1984; Turner, 1999) permitiendo explicar los acontecimientos de grupos a nivel intra e intergrupal, y cómo se afronta a nivel subjetivo el hecho de vivir con la enfermedad (Montiel \& Guerra, 2015).

\section{MÉTODO}

\subsection{Diseño}

Esta investigación se realizó en la ciudad de Cali, Colombia, durante todo el 2015. Se desarrolló bajo una perspectiva cualitativa explicativa con diseño narrativo, el cual permitió profundizar sobre el significado que los participantes le daban a "vivir con $\mathrm{VIH}^{\text {"; }}$ un momento de su historia que permitió dar cuenta al mismo tiempo de las percepciones de sí mismos y las construcciones que han hecho a partir de su experiencia de tener la enfermedad (Flick, 2004).

\subsection{Participantes}

Los participantes de los grupos de discusión fueron 16 personas, distribuidas en tres grupos. El primer grupo estuvo conformado por cuatro personas de género masculino entre los 30 y 49 años de edad; el segundo grupo lo conformaron siete personas de género femenino entre los 23 y 46 años de edad; y en el último grupo participaron cinco personas de género femenino entre los 22 y 44 años de edad. Todas las personas participantes vivían en la ciudad de Santiago de Cali, habían sido diagnosticadas con VIH/SIDA por transmisión sexual y asistían en ese momento a una organización no gubernamental que brinda apoyo psicoscosocial a personas con esta enfermedad (ver Tabla 1). 
Karen Macías Pulgarín, Paula Andrea Mendoza, Christian León Osorio, Fernando Riveros Munévar, Anderssen Vera, Arleth Patricia Bernal, Liliana Reyes

Tabla 1

Descripción sociodemográfica de los Participantes

\begin{tabular}{|c|c|c|c|c|c|c|c|c|c|c|}
\hline \multicolumn{2}{|c|}{ Participante } & Código & Grupo & Género & Edad & \multicolumn{3}{|c|}{ Tiempo de diagnóstico } & Grado & escolaridad \\
\hline & \multicolumn{2}{|c|}{ Estado civil } & \multicolumn{7}{|c|}{ Estado laboral } & \\
\hline 1 & P1 & 1 & M & 30 & 2 años & Octavo & Soltero & \multicolumn{2}{|c|}{ Empleado } & \\
\hline 2 & P2 & 1 & $M$ & 30 & 3 años & Técnico & \multicolumn{2}{|c|}{ Unión libre } & Desempleado & \\
\hline 3 & P3 & 1 & $M$ & 39 & 3 años & Once & \multicolumn{2}{|c|}{ Unión libre } & Independiente & \\
\hline 4 & P4 & 1 & $M$ & 49 & 1 año & Once & Soltero & \multicolumn{2}{|c|}{ Desempleado } & \\
\hline 5 & P5 & 1 & M & 33 & 2 años & Once & Soltera & \multicolumn{2}{|c|}{ Desempleado } & \\
\hline 6 & P6 & 2 & $\mathrm{~F}$ & 46 & 11 años & Once & Soltera & \multicolumn{2}{|c|}{ Desempleada } & \\
\hline 7 & P7 & 2 & $\mathrm{~F}$ & 38 & 1 año & Once & Casada & \multicolumn{2}{|c|}{ Empleada } & \\
\hline 8 & P8 & 2 & $\mathrm{~F}$ & 36 & 5 años & Once & Soltera & \multicolumn{2}{|c|}{ Empleada } & \\
\hline 9 & P9 & 2 & $\mathrm{~F}$ & 29 & 4 años & Quinto & \multicolumn{2}{|c|}{ Unión libre } & Desempleada & \\
\hline 10 & P10 & 2 & $\mathrm{~F}$ & 23 & 4 años & Cuarto & \multicolumn{2}{|c|}{ Unión libre } & Desempleada & \\
\hline 11 & P11 & 2 & $\mathrm{~F}$ & 40 & 5 años & Once & \multicolumn{2}{|c|}{ Unión libre } & Desempleada & \\
\hline 12 & P12 & 3 & $\mathrm{~F}$ & 38 & 13 años & Tercero & \multicolumn{2}{|c|}{ Unión libre } & Empleada & \\
\hline 13 & P13 & 3 & $\mathrm{~F}$ & 42 & 17 años & Once & Soltera & \multicolumn{2}{|c|}{ Independiente } & \\
\hline 14 & P14 & 3 & $\mathrm{~F}$ & 22 & 4 años & Once & \multicolumn{2}{|c|}{ Unión libre } & Independiente & \\
\hline 15 & P15 & 3 & $\mathrm{~F}$ & 38 & 3 años & Once & \multicolumn{2}{|c|}{ Unión libre } & Independiente & \\
\hline 16 & P16 & 3 & $\mathrm{~F}$ & 44 & 6 años & Once & \multicolumn{2}{|l|}{ Unión libre } & Empleada & \\
\hline
\end{tabular}

Los criterios de inclusión: A) Persona con diagnóstico confirmado de VIH/SIDA. B) Diagnóstico mayor o igual a seis meses. C) Personas con interés de participar voluntariamente en el estudio. D) Personas entre los 18 y 46 años. E) Firma del consentimiento informado. F) Haber participado en el estudio Fase 1 sobre Estigma Sentido y obtenido puntuaciones totales de estigma sentido. Los criterios de exclusión: A) Negación a firmar el consentimiento informado. B) Presencia de trastorno psiquiátrico o problemas en el desarrollo cognitivo que no permitan la comprensión de las actividades (indicado a través del personal de la ONG).

El tipo de muestreo que se utilizó para esta investigación fue por conveniencia. Este muestreo se caracterizó por la forma fácil y sin costo de acceder a la muestra (Mejía, 2000).

\subsection{Instrumentos}

La recolección de los datos se realizó a través de grupos de discusión liderados por las investigadoras del estudio utilizando una guía de preguntas orientadas a reconocer las experiencias de las personas de vivir con el diagnóstico de VIH. Para los grupos de discusión se utilizaron grabaciones de audio con autorización de cada una de los participantes, para su posterior transcripción y análisis. 


\section{Karen Macías Pulgarín, Paula Andrea Mendoza, Christian León Osorio, Fernando Riveros Munévar, Anderssen Vera, Arleth Patricia Bernal, Liliana Reyes}

Los grupos de discusión fueron transcritos de manera textual complementando con las notas de campo registradas durante las sesiones. Se realizó análisis temático, en el cual se determinaron conceptos en las respuestas de las personas con VIH, se clasificaron bajo las categorías establecidas y así se determinaron los significados, valoraciones y posiciones que jugaron un papel importante en las experiencias de los participantes (Flick, 2004) (ver Tabla 2).

\subsection{Procedimiento}

La presente investigación se desarrolló a través de las siguientes fases: a) Fase de acercamiento a la institución y contacto para el encuentro con los participantes. Se realizó el contacto con una de las organizaciones no gubernamentales [ONG] a las que asistían algunas de las personas que participaron en la Fase 1 del presente estudio (fase cuantitativa). Con el equipo psicosocial de la ONG se estableció la forma y los responsables de la invitación de los participantes a los grupos de discusión. La institución se encargó de hacer el contacto inicial con los posibles participantes y citar a una sesión informativa de la investigación. B) Fase de recolección de datos. En la sesión informativa con los participantes se les presentó la investigación: objetivos, procedimiento y consideraciones éticas. Posterior a la aceptación (todas las personas aceptaron participar) en los grupos de discusión, se establecieron las fechas de disponibilidad y se programaron las sesiones. Se realizaron tres grupos de discusión y se pidió autorización para grabar el audio, manteniendo la confidencialidad de la persona. Por tanto, en el audio no se registró ningún dato de identificación. Adicionalmente, se tomaron notas de campo, detallando el lenguaje no verbal. El grupo contó con preguntas orientadoras respecto a qué significa para ellos vivir con el $\mathrm{VIH}$. Posteriormente, se realizaron los análisis y presentación de los informes.

\subsection{Análisis de los datos}

Los datos fueron tratados por medio del programa Atlas Ti V6.0. Este software permitió desarrollar el análisis cualitativo, la organización, la reagrupación y la gestión de la información recogida con los participantes (datos textuales y gráficos). Se realizó un análisis temático, el cual se llevó a cabo, leyendo inicialmente el texto "fragmento por fragmento, para definir el contenido y las categorías" (Escalante \& Páramo, 2011, p.45), las cuales fueron inicialmente, construidas a la luz de los modelos orientadores de la presente investigación. Posteriormente, el análisis implicó: "frecuencia de aparición, variación según los hablantes, según los contextos, interdependencia entre los elementos de un modelo" (Escalante \& Páramo, 2011, p.45); tomando las investigadoras un rol importante, en la medida en que fueron quienes seleccionaron y construyeron a partir de los datos, mostrando su ideología e intención. 


\section{Karen Macías Pulgarín, Paula Andrea Mendoza, Christian León Osorio, Fernando Riveros Munévar, Anderssen Vera, Arleth Patricia Bernal, Liliana Reyes}

\subsection{Consideraciones éticas}

Esta investigación se enmarca en lo establecido en la Resolución No. 008430 de 1993 del Ministerio de Salud y la Ley 1090 del 6 de septiembre de $2006(42,43)$ por la cual se reglamenta el ejercicio de la profesión de Psicología. La presente investigación se ajusta a principios de beneficencia, no maleficencia y autonomía; y se clasificó como una INVESTIGACIÓN CON RIESGO MíNIMO. Los participantes que aceptaron hacer parte del estudio realizaron la firma del consentimiento informado.

\section{RESULTADOS}

Las personas participantes en el presente estudio fueron 16. Las edades estuvieron comprendidas entre los 22 y los 49 años de edad, y todos vivían actualmente en la ciudad de Santiago de Cali. La mayoría de ellos asistían a control en una institución de salud de la ciudad (tres personas refieren no asistir a los controles de forma voluntaria) y tenían entre 1 y 17 años de diagnóstico. Siete de los 16 participantes tienen dificultades de inserción laboral o no logran un trabajo que cumpla con las condiciones laborales legales (ver Tabla 1).

Las personas manifiestan por medio de sus narraciones que vivir con VIH ha generado diversas experiencias que han influido en la manera de ver y asumir la vida. Esto ha impactado la forma de percibirse a sí mismas y de resolver problemáticas relacionadas con la enfermedad. Las preguntas orientadoras del grupo facilitaron que contaran su experiencia desde el momento en que recibieron el diagnóstico y a lo largo de sus narraciones el tema de estigma social y sentido fue dominante. En ningún momento el equipo facilitador de los grupos insinuó el tema de estigma, ni orientó a los participantes para hablar sobre ello, pese a que habían sido invitados por haber obtenido niveles de estigma sentido en la Fase 1 del presente estudio.

A partir de la información analizada emergieron las siguientes categorías: estigma sentido, percepción de sí mismos y estrategias de afrontamiento. A continuación, se presentan los resultados obtenidos en cada una de las categorías (ver Tabla 2).

Tabla 2

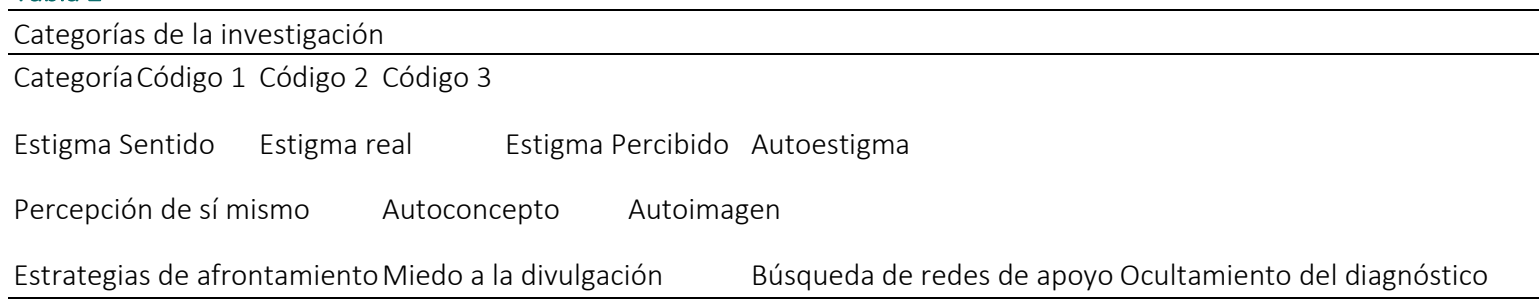

\subsection{Estigma Sentido}

A partir de las experiencias de los participantes se evidencia en sus narraciones que en un primer momento los participantes tenían ideas sociales del VIH construidas y relacionadas con el estigma sobre el diagnóstico de la enfermedad como sinónimo de muerte y como una condición biológica y poco factible de ser adquirida por ellos. Las personas refieren que, al recordar el momento de diagnóstico, en ningún momento se sintieron vulnerables o en riesgo de adquirir el $\mathrm{VIH}$. 


\section{Karen Macías Pulgarín, Paula Andrea Mendoza, Christian León Osorio, Fernando Riveros Munévar, Anderssen Vera, Arleth Patricia Bernal, Liliana Reyes}

“Cuando el doctor me dijo que tenía VIH se me vino el mundo encima, yo no sabía qué iba a hacer, yo recibí ayuda psicológica, pero no crea, eso fue muy duro para mí, yo sentía que si debía morirme, pues que me muriera y ya" (p.2).

"Yo pensaba que esto era imposible, yo pensaba que el VIH era muerte o un imposible y míreme ahora" (P7).

Los participantes expresan que han ocultado su enfermedad de manera pública y algunos lo han revelado a personas cercanas (familia nuclear, amigos y pareja), asimismo ellos manifiestan percibir de manera imaginaria que los demás sospechan que tienen el diagnóstico, que los comentarios que reciben tienen que ver con la enfermedad y tienden a anticipar sus comportamientos discriminatorios.

“...A veces mi hermana me hace comentarios sobre la salud y yo pienso que me los está diciendo a mí, he peleado con ella porque ando prevenido de que ella sepa porque ella sabe cosas sobre la salud" (p.3).

Es importante destacar que en los grupos de discusiones se presentaron verbalizaciones que hacían referencia a los conceptos subjetivos respecto a tener el VIH matizado por los prejuicios del contexto en el que se relacionan. Ellos expresan significados negativos y evitativos que dan cuenta de una representación de la enfermedad como un obstáculo en su vida que genera emociones relacionadas con la rabia, la tristeza, la angustia, y la culpa; además de desmotivación por vivir con VIH. Igualmente, las narraciones muestran cómo por parte de los profesionales de la salud se llevan ciertas acciones originadas en el diagnóstico que son discriminatorias.

"Yo siento que la gente a veces me discrimina por tener el VIH. Siento que los médicos más que todo a veces son los que lo ven a uno como menos. Una vez yo estaba en el ginecólogo haciéndome la citología y me dejaron de última, yo pregunte ¿Por qué? $Y$ me dijeron que porque tenía la enfermedad (llanto) yo siempre me acuerdo de eso" (p.12).

"Mire, pues yo no me tomo el medicamento, yo ahora no me lo tomo y no lo quiero tomar. Yo le llamo la "cosa" le prefiero decir así...todo el mundo ya sabía que yo tenía la enfermedad, la gente me decía cosas, me gritaban cosas y claro yo no me dejaba y les respondía, a veces me agarraba y bien feo" (p.10).

Se observan narrativas positivas sobre el vivir con $\mathrm{VIH}$; tres de las participantes de género femenino (una de ellas con el mayor tiempo de diagnóstico) expresaron que el VIH ha sido una experiencia que ha transformado su vida de manera que ha permitido en ellas transformarse como mujeres más allá del diagnóstico e incluso fue la oportunidad de desafiarse a sí mismas e ir resignificando su vida hacia una vida de mayor autosatisfacción. Incluso una de ellas refirió que esto le ha permitido asumir roles hacia el acompañamiento de otros con VIH o de disfrutar espacios como los grupos de apoyo de personas afectadas por el VIH/SIDA.

“...pero mire a pesar de eso yo me siento fuerte, yo siento que incluso he cogido cuerpo (risas) es que cuando me enfermé quedé como un palillo, ahora me siento fuerte, bonita, yo si voy a mi 


\section{Karen Macías Pulgarín, Paula Andrea Mendoza, Christian León Osorio, Fernando Riveros Munévar, Anderssen Vera, Arleth Patricia Bernal, Liliana Reyes}

médico, recojo mis medicamentos y pregunto todo lo que se me ocurre, yo les cuento lo que me pasa y donde no esté de acuerdo yo voy diciendo las cosas" (p.11).

\subsection{Percepción de sí misma(o)s}

Para todos los participantes la revelación del VIH ocasionó un impacto en ellos. La enfermedad a causa de su estigma social produce un cambio en la forma en que se veían antes y después de tener la enfermedad, en muchos de ellos la percepción que tienen de sí mismos está relacionada con la presencia o no de estigma sentido. El autoconcepto y la autoimagen de muchos de os participantes se han ido transformando teniendo en cuenta la manera en que perciben el VIH y las experiencias relacionadas con la enfermedad. Muchos de ellos sienten que por el hecho de tener la enfermedad pueden ser rechazados a nivel social y tienen dificultades para mostrarse como individuos pertenecientes a grupos con $\mathrm{VIH}$. Así mismo, manifiestan que existen personas que no entienden de la enfermedad y pueden llegar a juzgarlos, lo que puede convertirse en una experiencia angustiante y dolorosa. Se evidencia que la percepción de sí mismos que tienen los participantes ha dependido de la enfermedad, de lo que los demás digan de ellos, de lo que la familia opine de ellos, de sus ocupaciones, de su edad y de su ideología de vida, es decir, su autoconcepto y autoimagen ha sido construida por multicausal, siendo el VIH un aspecto transversal.

"Yo ahora es que estoy surgiendo entre las cenizas. Mi problema más importante no es el VIH. Yo fui consumidor de sustancias psicoactivas. No tengo una buena relación con mi familia...y yo quiero salir adelante, pero siempre hay algo que uno recuerda que lo hace a uno decaer; a mí me da duro que mis parejas me abandonen porque no sienten que conmigo tengan un futuro" (p.4).

"iAy mujeres!, yo si les digo que yo vivo muy feliz, yo no sé qué me pasa a mí. Yo como que no me acuerdo que tengo esta enfermedad. No sé si es por el tiempo que llevo, yo vivo feliz de la vida. Incluso yo me volví fue más fuerte, empecé a salir más, tengo hartísimas amigas, hay yo aprendo cada día tantas cosas; mire, esto me hizo ver que yo no me podía quedar sin hacer nada y viera que empecé a entender que no podía quedarme así solamente sin hacer nada por la vida, que nadie me iba a hacer siempre las cosas y me iba a mantener; entonces yo cogí y empecé a buscar por mis lados cómo trabajar, empecé a salir, conocer gente y soy muy feliz. Yo me siento muy bonita, muy importante (risas). Y el VIH ni me acuerdo de eso" (p.8).

"Yo en lo personal pues he tenido una vida de subidas y bajadas. Yo tengo una hija ahora y pues tengo tantas cosas por qué preocuparme, el $\mathrm{VIH}$, tratar de sobrevivir en el barrio y tener con qué comer (llanto)" (p.9).

"Yo siento que uno va madurando en el tema cuando avanza la enfermedad, como cuando ya llevas mucho tiempo con la enfermedad. A mí al principio los médicos me dijeron que me iba a morir por la enfermedad y me dejaba guiar mucho por lo que me decía la gente o lo que pensaban los demás, ya ahora, no, pues no me importa lo que diga la gente, si me quieren así bien, sino chao" (p.13).

"Yo he cambiado mucho, antes era un poco más sociable ahora me volví un poco más introvertida, la verdad me gustaría volver a recuperar mi forma de ser. Por parte de mi familia he recibido unos desplantes que me han hecho sentir un poco mal" (p.15). 


\section{Karen Macías Pulgarín, Paula Andrea Mendoza, Christian León Osorio, Fernando Riveros Munévar, Anderssen Vera, Arleth Patricia Bernal, Liliana Reyes}

\subsection{Estrategias de afrontamiento}

EI VIH como enfermedad de características sociales, en los participantes del presente estudio tuvo influencias en la manera cómo recibían el diagnóstico y así mismo cómo lo afrontaban. Sus estrategias de afrontamiento estuvieron matizadas por la manera en que había sido revelado el diagnóstico y de la percepción de confianza con su familia. Así mismo, el significado que le daban a la enfermedad dependía de la manera en que la afrontaban.

Se puede evidenciar que existe la estrategia de búsqueda de apoyo social, el cual puede ser familiar o de amigos, lo cual les ayuda a los individuos a sentirse acompañados, escuchados y comprendidos respecto a su enfermedad:

"Yo he aprendido a vivir con el VIH porque tengo el apoyo de mi madre gracias a Dios. Al principio a mí me dio bien duro; pensé que iba a morir ahí mismo, pero a uno los doctores le empiezan a explicar que uno realmente no se va a morir. Yo aquí en la fundación he conocido gente que tiene el diagnóstico y uno aprende de ellos ¿si me entiende?, eso le ayuda a uno mucho" (p.1).

"A mí me revelaron muy bien el diagnóstico gracias a Dios, tuve la oportunidad de tener buenos especialistas que me explicaron todo muy a detalle y me escuchaban más allá de cualquier cosa; eso me ayudó muchísimo" (p.16).

Existe la estrategia de afrontamiento que esta direccionada hacia el ocultamiento de la enfermedad como mecanismo de defensa para que las personas no generen prejuicios y así mismo no se presente un malestar emocional. En este caso, la percepción de lo que los demás piensan del individuo es de gran importancia y revela también que pese al tiempo de diagnóstico existen aún desconocimiento sobre las formas de transmisión o propagación de la infección.

“Mi familia pues tampoco sabe que tengo VIH porque a mí me da miedo perderlos, sobre todo a mis sobrinas, que mi hermana que es enfermera piense que yo les voy a pegar la enfermedad o algo así y pierda el cariño de todos ellos" (p.3).

También se puede destacar que en los participantes se evidenciaba como estrategia de afrontamiento centrada en la emoción, el miedo. Este se da como una respuesta para protegerse de personas que pueden tener conductas despectivas sobre el estado serológico. El miedo en los participantes también se puede relacionar con la percepción que tienen de sí mismos y haber sido estigmatizado por tener el $\mathrm{VIH}$. Igualmente, se puede evidenciar cómo en las narrativas de las personas se resalta nuevamente el desconocimiento sobre la transmisión del virus y la importancia del tratamiento farmacológico y no farmacológico.

"Yo no quiero encontrarme a nadie conocido cuando vaya al médico, yo vivo en un barrio donde la gente es ignorante, una vez se enteraron que una persona tenía VIH y la apedrearon (llanto) (ocultamiento). Yo no siento que necesite el medicamento y pues no iré, a mí no me gusta estar contando cosas de mi vida personal, o sea, para mí no es fácil estar contando cosas de mi vida a personas que no sé si van a contar cosas mías; yo no soy así" (P9). 


\section{Karen Macías Pulgarín, Paula Andrea Mendoza, Christian León Osorio, Fernando Riveros Munévar, Anderssen Vera, Arleth Patricia Bernal, Liliana Reyes}

\section{DISCUSIÓN}

El estigma sentido es un aspecto importante a considerar a la hora de abordar integralmente a una persona con VIH/SIDA. Investigaciones previas demuestran la importancia y el alto porcentaje de estigma sentido que tienen las personas con $\mathrm{VIH}$, así como el impacto a nivel biopsicosocial (Almanza \& Flores, 2012; Leserman, Barroso, Pence, Salahuddin \& Harmone, 2008; Moral \& Segovia, 2011; Saa, 2008; Schuster, Bornovalova \& Hunt, 2011; Varas, Neilands, Guilamo \& Cintron, 2008), por ejemplo, el impacto que el estigma sentido tiene en la adherencia al tratamiento, el recibir y solicitar apoyo social, en la revelación del diagnóstico, en las interacciones sociales y situaciones de participación social. Es frecuente (Sontag, 2003) que personas que tienen estigma sentido se aíslen, oculten su diagnóstico, se abstengan de tener relaciones sexuales, tengan una baja adherencia al tratamiento y presenten mayores niveles de ansiedad, depresión y estrés.

La presente investigación hace relevancia a considerar el VIH no solo como una enfermedad de componente biológico e intrapsicológico sino que es necesario identificar el aspecto social al que se liga porque la construcción de la imagen social de una enfermedad puede definir de forma importante, cómo una persona se posiciona en su espacio social, se percibe a sí misma y con qué individuos de su comunidad se identifica, es decir, si una persona se identifica con un grupo que ha sido marginado y una enfermedad que ha sido estigmatizada es consecuente que vaya a tener un autoconcepto construido desde la enfermedad y no desde sus experiencias subjetivas y que esto a su vez implique consecuencias a nivel psicológico como baja autoestima, rechazo a la enfermedad, negación de la enfermedad, falta de adherencia al tratamiento y dificultades para establecer relaciones interpersonales.

Teniendo en cuenta lo anterior, los hallazgos de la presente investigación permiten evidenciar que el VIH es producto de diversas situaciones en cadena, en donde las representaciones sociales construidas alrededor de la enfermedad son un factor central en el afrontamiento y en la forma de vivirlo colectivamente. Indudablemente, el VIH es una enfermedad de gran carga social, que genera frecuentemente comportamientos de rechazo, distanciamiento, señalamiento hacia quienes tienen el diagnóstico, e incluso entre las mismas personas que viven con la infección.

Se encuentra por medio de este estudio que son diversos los aspectos que han impactado en la representación social del VIH. Los resultados de esta investigación evidencian que existen aún muchos interrogantes respecto a la etiología de la enfermedad y esto genera una distorsión en la imagen que las personas tienen de la enfermedad y por consecuente de sí mismas, porque la carga social y del grupo con el que se identifican $(\mathrm{VIH})$ es un grupo que ha sido caracterizado históricasocialmente desde lo "negativo". Se encontró que las personas al identificarse con "ideas" producto del estigma social, se autoperciben con características subjetivas negativas (Hay una individuación negativa).

Los resultados encontrados en la presente investigación permiten conocer que a nivel colectivo las personas con VIH se identifican endogrupalmente con personas que tienen su misma enfermedad pero son los tabúes y mitos de la enfermedad (también se asocia el VIH a grupos que han sido 


\section{Karen Macías Pulgarín, Paula Andrea Mendoza, Christian León Osorio, Fernando Riveros Munévar, Anderssen Vera, Arleth Patricia Bernal, Liliana Reyes}

segregados como hombres que se relaciona sexualmente con otros hombres, trabajadores sexuales, transgeneristas, consumidores de drogas inyectables) lo que hace que se identifiquen negativamente y que en la mayoría de los casos haya negación y ocultamiento de la enfermedad. El resultado de esta identificación colectiva es que muchas veces las personas con VIH se aíslan para que nadie conozca su diagnóstico y así mismo eviten acceder a lugares públicos de salud y a los tratamientos que evitan un deterioro del sistema inmunológico.

A nivel subjetivo se encontró que las personas con VIH categorizan su "Yo" no solamente desde su individualidad, su historia de vida (antes de adquirir el VIH) y características propias, sino que a partir de que una persona se entera de su diagnóstico (si no recibe un apoyo psicosocial adecuado) hay una ruptura en el concepto que tenía de sí mismo, pasando a considerarse lleno de características negativas construidas a nivel social. A partir de este postulado y de los resultados encontrados se logró encontrar una relación entre la autopercepción negativa y la culpa que algunas de las personas pueden llegar a tener. En este sentido, se logró conocer que las personas con VIH que tenían una imagen negativa de la enfermedad se sentían al mismo tiempo culpables debido a la carga emocional a nivel social que representa. Es, así pues, que las personas con VIH al recibir su diagnóstico pueden tener una ruptura a nivel subjetivo (autopercepción) que es la que genera al mismo tiempo la autodiscriminación y el estigma sentido.

A partir de lo postulado, también se plantea que la atención en salud es clave para la aceptación y manejo que hace la persona de su enfermedad y su situación de salud. Indudablemente, ese momento es fundamental para el afrontamiento y adaptación a la enfermedad, al tratamiento y a las dinámicas propias que emergerán en los estilos de vida. Además, es necesario que los profesionales que trabajan con el VIH, aborden sobre cómo los individuos con la enfermedad han construido la imagen de sí mismos y cómo esto se puede transformar en un autoconcepto negativo para generar intervenciones orientadas a la resiliencia y a un afrontamiento propositivo y proactivo.

Los retos para el abordaje del VIH entre las ciencias de la salud y las ciencias sociales son grandes. Es claro que, el VIH hace necesario el abordaje integral, en donde no solo se trabaje el paciente, sino también en el acompañamiento a las familias, la red de apoyo de estas personas e incluso en personas que no tengan la enfermedad, todo lo relacionado con el estigma social, la discriminación y el estigma sentido. De esta manera, se reconoce la necesidad de hacer un abordaje biopsicosocial respecto a esta temática en particular.

Es posible hacer referencia a que las intervenciones para trabajar el estigma sentido aún siguen siendo escasas; o han tenido en su metodología una secuencia de similitudes para abordar el tema, sin muestras de gran efectividad a mediano y largo plazo. Las intervenciones en estigma sentido han estado orientadas a ofrecer a los participantes información respecto a la salud, el liderazgo, estrategias de afrontamiento y derechos (Nyamathi et al., 2013); además de educación respecto al estigma, entrenamiento en relajación, entrenamiento en afrontamiento, juego de roles, resolución de problemas (Rao et al., 2012).

Estas formas de intervenir, a pesar de ser muy importantes y sólidas, en personas con alto grado de estigma sentido también han generado brechas que ocasionan un estilo reducido para abordar esta 


\section{Karen Macías Pulgarín, Paula Andrea Mendoza, Christian León Osorio, Fernando Riveros Munévar, Anderssen Vera, Arleth Patricia Bernal, Liliana Reyes}

temática, no integrando perspectivas interdisciplinares para hacer referencia a una condición tan compleja como lo es el estigma sentido en personas con VIH. Cuando se hace referencia a la autodiscriminación y autopercepción de un grupo de personas que tienen una enfermedad con tantas incógnitas y sesgos sociales, se deben tener en cuenta aspectos a abordar como la construcción de su subjetividad e identidad colectiva para lograr encontrar formas de afrontar esa herida social.

De esta manera, la presente investigación a partir de las aproximaciones al estigma social y al estigma sentido desde las narraciones de las personas con $\mathrm{VIH}$, considera que una intervención en el contexto colombiano es necesaria. Los elementos claves para una posible intervención son la reconstrucción de la historia del VIH, la memoria colectiva y la identidad colectiva. En este sentido, se plantea la narrativa autobiográfica como estrategia para hacer consciencia de lo que una persona con VIH piensa de sí mismo y acompañar hacia una diferenciación entre el estigma percibido o el estigma real en el grupo de personas a intervenir y que cada uno logre observar las características que lo definen más allá de sus condiciones de vida específicas.

Por otro lado, realizar una intervención grupal contribuiría a fomentar el vínculo colectivo, lo cual podría permitir beneficios en la cohesión grupal y social para movilizar motivaciones que permitan crear estrategias propias para afirmar su identidad desde lo que son una vez que se identifiquen con el grupo más allá de la enfermedad (Bruner, 1991; Goffman, 1963). En este orden de ideas, las intervenciones podrían direccionarse a reconstruir y potencializar el concepto que la persona tiene de la enfermedad y respecto a sí mismo (individual y grupalmente) para que puedan tener una visión no de culpabilidad sino de responsabilidad personal y colectiva, lo que permitirá que la enfermedad se valore de una manera diferente (Collins, 2009; Tajfel, 1984).

Igualmente, se requiere que estas intervenciones aborden el componente colectivo, educativo y sociocultural, que haya una comprensión histórica sobre la aparición del estigma social hacia el VIH y el verdadero origen del $\mathrm{VIH}$, para posteriormente realizar actividades de educación y comunicación en salud que tengan impacto en los diferentes grupos, poblaciones y difuminen las fronteras sociales que parecieran existir entre quien tiene una enfermedad y quien no la tiene. Esto es central para la disminución del estigma social y por tanto, en la construcción de representaciones sociales propositivas, científicas y humanizantes respecto al VIH que al mismo tiempo disminuyan el estigma sentido.

Nota de Autores

Artículo de investigación producto del estudio titulado Estigma sentido en población colombiana y puertorriqueña con VIH/SIDA - Fase 2, código: 3958. 


\section{Karen Macías Pulgarín, Paula Andrea Mendoza, Christian León Osorio, Fernando Riveros Munévar, Anderssen Vera, Arleth Patricia Bernal, Liliana Reyes}

\section{REFERENCIAS}

Almanza, A. \& Flores, F. (2012). Resistencia a la discriminación: Narrativas familiares acerca de la infección por VIH. Un estudio exploratorio. Psicología y Salud, 22(2), 173-184. Dsiponible en https://www.uv.mx/psicysalud/psicysalud-22-2/22-2/Ariagor\%20Almanza\%20Avenda\%F1o.pdf

Bruner, J. (1991). Actos de significado: más allá de la revolución cognitiva. Madrid: Alianza 1.

Collins, R. (2009). Cadenas de rituales de interacción. Barcelona: Arthropos Editorial.

Escalante, E. \& Páramo, M. (2011). Aproximación al análisis de datos cualitativos: aplicación en la práctica investigativa. 1a. ed. Mendoza: Universidad del Aconcagua.

Flick, U. (2004). Introducción a la investigación cualitativa. Madrid: Ediciones Morata.

Gallo, R. \& Montagnier, L. (2004). El descubrimiento del VIH como causa de sida. Revista del Hospital Materno Infantil Ramón Sardá, 23(2), 88-91. Dsiponible en http://www.scielo.org.mx/scielo.php?script=sci_arttext\&pid=S0034-83762004000200003

Goffman, E. (1963). Stigma: notes on the management of spoiled identity. New York: Prentice-Hall.

Hoyos-Hernández, P. A. \& Duarte-Alarcón, C. (2016). Roles y desafíos de mujeres jefas de hogar con VIH/Sida. Revista de Salud Pública, 18(4), 554- 567. http://dx.doi.org/10.15446/rsap. v18n4.42096

Jiménez, J., Puig, M., Ramos, J., Morales, M., Asencio, G., Sala, A., Castro, E., Vélez, C., Santiago, L. \& Zorrilla, C. (2010). Measuring HIV felt stigma: A culturally adapted scale targeting PLWH in Puerto Rico. AIDS Care, 22(11), 13141322. DOI: $10.1080 / 09540121003758481$

Jiménez, J., Morales, M., Castro, E., Puig, M., Vélez, CN., Santiago, L. \& Zorrilla, C. (2012). Levels of Felt Stigma among a Group of People with HIV in Puerto Rico. Puerto Rico Health Sciences Journal, 31(2), 64-70. Dsiponible en https://www.ncbi.nlm.nih.gov/pubmed/22783698

Leserman, J., Barroso, J., Pence, B., Salahuddin, N. \& Harmone, J. (2008). Trauma, stressful life events and depression predict HIV-related fatigue. Aids care, 20(10), 1258-65. https://doi.org/10.1080/09540120801919410

Mejía, J. (2000). El muestreo en la investigación cualitativa. Investigaciones Sociales, 4(5), 165-180. Ministerio de Salud (2006). Resolución № $008430 \quad 1993 . \quad$ Recuperado de http://www.dib.unal.edu.co/promocion/etica_res_8430_1993.pdf

Miric, M. (2004). Escala para la evaluación del estigma sentido entre las personas VIH positivas en la República Dominicana. Paradigmas, 4(5), 70-101. Dsiponible en http://pepsic.bvsalud.org/pdf/pp/v5/v5a06.pdf

Montiel, V. \& Guerra, V. (2015). Aproximaciones teó- ricas sobre psicoeducación. Análisis de su aplicación en mujeres con cáncer de mama. Psicogente, 19(36), 324-335). http://doi.org/10.17081/psico.19.36.1301

Moral, J. \& Segovia, M. (2011). Discriminación en mujeres que viven con VIH/SIDA. Revista Iberoamericana de Psicología y Salud, 2(2), 185-206. Dsiponible en http://www.redalyc.org/articulo.oa?id=245118507004

Moral, J. \& Segovia, M. (2015). Discriminación en la familia a mujeres que viven con VIH. Psicogente, 18(33), 89-103. http://doi.org/10.17081/psico.18.33.58 


\section{Karen Macías Pulgarín, Paula Andrea Mendoza, Christian León Osorio, Fernando Riveros Munévar, Anderssen Vera, Arleth Patricia Bernal, Liliana Reyes}

Nyamathi, A., Ekstrand, M., Salem, B., Sinha, S., Ganguly, K., Leake, B. \& Marfisee, M. (2013). Impact of Asha intervention on stigma among rural Indian women with AIDS. Western Journal Nursing Res, 35(7), 867-883. DOI: 10.1177/0193945913482050

Organización Mundial de la Salud (2011). VIH/sida. Centro de Prensa (Nota descriptiva N 360). Recuperado de http://www.who.int/mediacentre/factsheets/fs360/es/

Rao, D., Desmond, M., Andrasik, M., Raberry, T., Lambert, N., Cohn, S. \& Simoni, J. (2012). Feasibility, acceptability, and preliminary efficacy of the unity workshop: An internalized stigma reduction intervention for African American women living with HIV. AIDS patient care and STDs, 25(10), 614- 620. https://doi.org/10.1089/apc.2012.0106

Saa, D. (2008). Relación médico paciente. Colombia Médica, 9(3), 287-90. Dsiponible en http://scielo.sld.cu/scielo.php?script=sci_arttext\&pid=S0864-34662006000400007

Schuster, R., Bornovalova, M. \& Hunt, B. (2011). The influence of depression on the progression of HIV: direct and indirect effects. Behavior Modification, 36(2), 123-45. https://doi.org/10.1177/0145445511425231

Sontag, S. (2003). La enfermedad y sus metáforas/el sida y sus metáforas. Buenos Aires: Taurus.

Tajfel, H. (1984). Grupos humanos y categorías sociales. Barcelona: Herder.

Turner, J.C. (1999). Some current issues in research on social identity and selfcategorization theories. In N. Ellemers, R. Spears \& B. Doosje (Dir), Social identity: Conext, commitment, content (pp. 6-34). Oxford: Basil Blackwell.

Varas, N., Neilands, B., Guilamo, V. \& Cintron, F. (2008). Desarrollo de la Escala sobre Estigma Relacionado con el VIH/SIDA para Profesionales de la Salud mediante el Uso de Métodos Mixtos. Revista Puertorriqueña de Psicología, 19, 183-215. Dsiponible en https://www.ncbi.nlm.nih.gov/pmc/articles/PMC2843427/

Está obra está bajo: Creative commons attribution 4.0 international license. El beneficiario de la licencia tiene el derecho de copiar, distribuir, exhibir y representar la obra y hacer obras derivadas siempre y cuando reconozca y cite la obra de la forma especificada por el autor o el licenciante

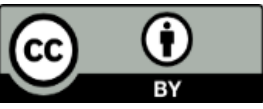

